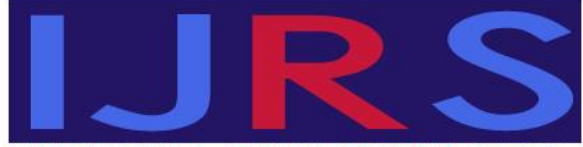

. INTERNATIONAL JOURNAL OF ROMA STUDIES.
Hipatia Press

www.hipatiapress.com

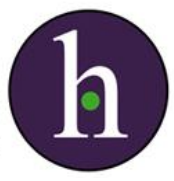

Instructions for authors, subscriptions and further details:

http://ijrs.hipatiapress.com

\title{
Un Paso Más en la Investigación en Trabajo Social: Aportaciones de la Metodología Comunicativa
}

Teresa Plaja Viñas ${ }^{1}$

1) University of Barcelona. Spain

Date of publication: September $15^{\text {th }}, 2019$

Edition period: September 2019 - March 2020

To cite this article: Plaja Viñas, T. (2019). Un paso más en la investigación en Trabajo Social: aportaciones de la Metodología Comunicativa.

International Journal of Roma Studies, 1(2), 144-158. doi:

10.17583/ijrs.2019.4312

To link this article: http://dx.doi.org/10.17583/ijrs.2019.4312

\section{PLEASE SCROLL DOWN FOR ARTICLE}

The terms and conditions of use are related to the Open Journal System and to Creative Commons Attribution License (CCAL). 


\section{One More Step in Social Work Research: Contributions of the Communicative Methodology}

Teresa Plaja Viñas

University of Barcelona

\section{Abstract}

Since the beginning of Social Work, the importance of research as a tool to achieve its objective has been stressed: social change based on the defense of human rights and social justice. However, a gap between research and professional practice persists. Given this situation that limits its capacity for transformation, Social Work has not remained static, but has sought ways to overcome it. This article presents the Communicative Methodology and its already demonstrated social impact, as a way to contribute to the reduction of the gap between research and practice. Although the first study based on it focused on the Roma people, it is currently being carried out in almost all the contexts in which Social Work intervenes. Given the importance of respecting the principles of Social Work to achieve its objectives, the article explores the fit of the Communicative Methodology in research in this discipline. For this, the principles and objectives of Social Work established by the IFSW are related to those of this methodology.

Keywords: Research; Social work; Communicative Methodology; Social impact 


\section{Un Paso Más en la Investigación en Trabajo Social: Aportaciones de la Metodología Comunicativa}

Teresa Plaja Viñas

Universitat de Barcelona

\section{Resumen}

Desde los inicios del Trabajo Social se ha subrayado la importancia de la investigación como herramienta para alcanzar su objetivo: el cambio social en base a la defensa de los derechos humanos y la justicia social. No obstante, persiste una brecha entre la investigación y la práctica profesional. Ante esta situación que limita su capacidad de transformación, el Trabajo Social no se ha quedado estático, sino que ha buscado vías para superarlo. Este artículo presenta la Metodología Comunicativa y su ya demostrado impacto social, como una vía para contribuir a la reducción de la brecha entre la investigación y la práctica. Aunque el primer estudio basado en ella se centró en el pueblo gitano, en la actualidad se desarrolla ya en casi todos los contextos en los que interviene el Trabajo Social. Ante la importancia de respetar los principios del Trabajo Social para alcanzar sus objetivos, el artículo explora el encaje de la Metodología Comunicativa en la investigación en esta disciplina. Para ello se ponen en relación los principios y objetivos del Trabajo Social establecidos por la IFSW con los de esta metodología.

Palabras clave: Investigación; Trabajo Social; Metodología Comunicativa; Impacto social 


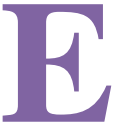

n 1910, Jane Addams publicó su libro "Veinte años en Hull House" que exponía la importancia de realizar investigaciones antes de iniciar nuevas actividades con el fin de ofrecer respuestas adecuadas a las necesidades a las que debía hacer frente la comunidad (Addams, 2014). En esta línea, en 1917, Mary Richmond publicó "Diagnóstico Social". En él, a partir de una exhaustiva investigación que tenía como objetivo mejorar la práctica profesional de los y las trabajadoras sociales, la autora presenta el método para elaborar el diagnóstico social en Trabajo Social (TS a partir de ahora) (Richmond, 2005). En este mismo libro, Richmond exponía la importancia de la recopilación de evidencias procedentes de diferentes fuentes para establecer un diagnóstico social con el objetivo de marcar el plan de intervención (Richmond, 2005).

Sin embargo, pese a la importancia que desde los orígenes del TS se ha dado a la investigación para orientar la práctica profesional y a que la investigación forma parte del procedimiento metodológico del TS, en la actualidad persiste una brecha entre la investigación y la práctica profesional (Gray, Sharland, Heinsch, \& Schubert, 2015; Rubin, 2015). Esta brecha limita las posibilidades del TS para alcanzar su objetivo de manera efectiva: el cambio social en base a la defensa de los derechos humanos y la justicia social (IFSW, 2014).

Concretamente, esta brecha puede implicar, por un lado, una restricción en el impacto social que alcanza la investigación en TS y, por otro lado, limitaciones en la práctica profesional al no incorporar de manera sistematizada el conocimiento y las evidencias científicas para así aumentar y garantizar la efectividad de las intervenciones. Con respecto al primer impedimento, se está exigiendo que la investigación tenga impacto social en todas las áreas de la ciencia, con el fin de contribuir a alcanzar los objetivos establecidos por la sociedad, como los Objetivos de Desarrollo Sostenible marcados desde las Naciones Unidas (United Nations, 2015). La importancia de alcanzar impacto social en la investigación se vio reflejada en el cuestionamiento de la financiación de la investigación en ciencias sociales y humanidades en los Programa Marco de la Unión Europea por su bajo impacto social (R. Flecha, Soler, \& Sordé, 2015; Sordé \& Mertens, 2014). Asimismo, en relación a la segunda limitación, es necesario conocer y utilizar en la práctica profesional herramientas basadas en evidencias 
científicas para garantizar la consecución de los objetivos de cambio social planteados en la intervención del TS (Munté Pascual \& Vicente Zueras, 2012).

Ante esta realidad, el TS ha desarrollado estrategias para reducir dicha brecha (Rubin, 2014) y, de este modo, realizar investigaciones con un mayor impacto social y aumentar la efectividad de sus intervenciones. Entre las estrategias desarrolladas encontramos la Evidence Based Practice (Thyer, 2010) o la creación de estructuras de colaboración entre las organizaciones de servicios sociales y la universidad (Steens, Van Regenmortel, \& Hermans, 2018).

También con el objetivo de reducir la brecha entre la investigación y la práctica, Pulido y Munté (2014) exponían la importancia para el TS de conocer las Actuaciones de Éxito identificadas y descritas por la comunidad científica internacional para ofrecer respuestas efectivas a las necesidades de las comunidades y colectivos con los que trabajan los y las profesionales. Las Actuaciones de Éxito son acciones basadas en evidencias científicas que permiten obtener resultados excelentes independientemente del contexto en el que se apliquen (R. Flecha, 2015), tienen un carácter universal y transferible (Rios, 2013).

Las Actuaciones de Éxito fueron identificadas y analizadas por el proyecto INCLUD-ED. Strategies for inclusion and social cohesion in Europe from Education financiado por el sexto programa Marco de Investigación de la Comisión Europea y tenía como objetivo identificar estrategias educativas que reducen las desigualdades sociales en los ámbitos de salud, empleo, vivienda y participación. El proyecto INCLUD-ED utilizó la Metodología Comunicativa (MC en adelante) para realizar su investigación, alcanzando un gran impacto social en diferentes ámbitos y colectivos (Brown, Gómez González, \& Munté Pascual, 2013; Garcia Yeste, Gairal Casado, Munte Pascual, \& Plaja Viñas, 2018; Melgar, 2015). Dicho impacto se evidenció en la selección del proyecto INCLUD-ED como el único de ciencias sociales y humanidades en la lista que la Comisión Europea publicó de los 10 proyectos con éxito de los Programas Marco de Investigación de la Comisión Europea (European Commission, 2011). 
La MC ha posibilitado realizar investigaciones con un alto impacto a nivel político, social y/o científico en ámbitos como la educación (Garcia Yeste, Morlà Folch, \& Ionescu, 2018), la prevención de la radicalización (Aiello, Puigvert, \& Schubert, 2018), la violencia de género (Valls, Puigvert, Melgar, \& Garcia-Yeste, 2016) o con la comunidad gitana (Munte, Serradell, \& Sorde, 2011). En relación a este último ámbito, fue en el proyecto WORKALÓ. The creation of new occupational patterns for cultural minorities: The Gypsy case que se utilizó la MC por primera vez, siendo su uso una de las principales aportaciones del proyecto (Sordé, $\mathrm{R}$. Flecha, \& Mircea Alexiu, 2013). WORKALÓ que tenía como objetivo definir estrategias de desarrollo social y económico orientadas a la cohesión social, poniendo especial atención a la comunidad gitana (WORKALÓ, 2001-2004), alcanzó un gran impacto político a nivel europeo y estatal (Macías \& Redondo, 2012; Sordé et al., 2013).

A raíz de las investigaciones que se han realizado utilizando la MC en los diferentes ámbitos expuestos y su gran impacto, esta metodología se dibuja como una vía para realizar investigaciones que contribuyan a reducir la brecha entre investigación y práctica en TS. Sin embargo, no solo es necesario centrarnos en realizar investigaciones que tengan impacto social, sino también que respeten los principios del TS. Esta necesidad queda evidenciada ante las investigaciones que, a lo largo de la historia y desde diferentes ámbitos, se han realizado sobre la comunidad gitana, pero sin la comunidad gitana. En algunas ocasiones estas investigaciones han contribuido a perpetuar las situaciones de desigualdad que vive esta comunidad (Macías \& Redondo, 2012; Munte et al., 2011; Sordé et al., 2013).

Teniendo en consideración la importancia que la investigación contribuya a alcanzar los objetivos del TS respetando sus principios, se evidencia la relevancia de que en la investigación no solo se disponga de metodologías que contribuyan a superar la brecha entre investigación y práctica, sino que además compartan dichos principios y objetivos. Con el fin de dar respuesta a esta necesidad, el presente artículo analiza el encaje de la MC con el TS, poniendo en relación los principios y los objetivos de la MC con los del TS expuestos por la International Federation of Social Workers (IFSW), la definición de TS más aceptada y reconocida a nivel 
internacional (M. Á. Pulido \& Munté, 2014) y con la Declaración Global de los Principios Éticos del Trabajo Social aprobada en 2018.

\section{Metodología}

Para dar respuesta al objetivo planteado, en primer lugar, se ha realizado un análisis documental de artículos científicos publicados en las principales bases de datos como Web of Science y SCOPUS, así como libros especialmente relevantes en la temática de estudio. Concretamente, se ha examinado literatura científica que analiza la MC, el uso de evidencias científicas en la práctica profesional en TS y el impacto social de la investigación en ciencias sociales.

Posteriormente, se han puesto en relación los elementos que caracterizan y definen la MC identificados en la literatura científica con los principios y objetivos expuestos en la Declaración Global de los Principios Éticos del TS aprobada en 2018 y la definición de TS aprobada por la International Federation of Social Workers (IFSW) y la International Association of Schools of Social Work (IASSW) en julio de 2014. En dicha definición se desglosan los conceptos que recoge la definición de TS, detallando sus deberes básicos, principios, conocimientos y práctica profesional (IFSW, 2014).

\section{Resultados}

A partir del análisis realizado, se han identificado elementos compartidos entre el TS y la MC, tanto en los objetivos que persiguen como en sus principios. Esta metodología se asienta en siete postulados en los que se tienen en consideración las aportaciones teóricas de autores como Habermas, Beck, Freire o Vygotsky (A. Gómez, Padrós, Ríos, Mara, \& Pukepuke, 2019; A. Gómez \& Díez-Palomar, 2009; J. Gómez, Latorre, Sánchez, \& R. Flecha, 2006) y que se incorporan a partir de la organización comunicativa de la investigación (A. Gómez et al., 2019)

La MC se sitúa dentro del paradigma comunicativo (Gómez, Puigvert, \& R. Flecha, 2011; Puigvert, 2012). Esta metodología incluye la voz de los colectivos investigados, no como objetivo en sí mismo sino como vía para 
superar las situaciones de desigualdad y exclusión que viven a partir del rigor científico y la utilidad social de la investigación (Valls \& Padrós, 2011). Para ello, se analizan tanto los factores que perpetúan las situaciones de desigualdad y exclusión social como aquellos que las transforman ( $R$. Flecha García \& Soler, 2013). De esta manera, se presta especial atención al impacto social de la investigación tanto durante el proyecto como una vez finalizado (R. Flecha \& Soler, 2014; J. Gómez et al., 2006).

En el presente artículo se destacan tres elementos identificados que evidencian la concordancia de los objetivos y principios de la MC con el TS. El primer elemento identificado hace referencia a su objetivo, concretamente a su orientación al cambio social. A continuación, se expone el segundo elemento común: la identificación de elementos exclusores y transformadores. Finalmente, el tercer elemento identificado hace referencia a la organización de la investigación, con el objetivo de garantizar la participación de la comunidad.

\section{Orientación al cambio social}

El TS orienta su práctica profesional y su investigación al cambio social a partir de la defensa y promoción de los derechos humanos y la justicia social, por lo que sus investigaciones y teorías son aplicadas y emancipadoras (IFSW, 2014; IFSW, 2018). Este objetivo es compartido con la MC (A. Gómez et al., 2019), la cual "persigue una utilidad social concreta: la transformación de las desigualdades sociales" (R. Flecha, Vargas, \& Dávila, 2004, p. 24).

Tal como se ha expuesto anteriormente, el proyecto INCLUD-ED permitió identificar cómo la exclusión educativa afecta a los ámbitos de salud, vivienda, trabajo y participación política. Sin embargo, INCLUD-ED no se limitó a elaborar un diagnóstico de la realidad, sino que identificó y analizó aquellas actuaciones que permitían superar dichas situaciones (Aubert, 2011).

Esta orientación al cambio social hace que la MC no sea una lista de métodos y técnicas de investigación, sino que a partir del compromiso con la justicia social y la participación de todos los colectivos y personas implicadas permite realizar investigaciones orientadas a la transformación 
social (A. Gómez \& Munté, 2015). Esta metodología permite, por un lado, buscar acciones que ya han evidenciado ser efectivas, identificando aquellos principios universales que posibilitan dicho éxito y que pueden adaptarse a otros colectivos y contextos en los que deben enfrentarse desafíos similares (R. Flecha \& Soler, 2014). Y por el otro lado, diseñar nuevas acciones basadas en evidencias que permiten superar las situaciones de desigualdad y exclusión social, es decir, generar creación social (Aiello \& Joanpere, 2014).

\section{Identificación de elementos exclusores y transformadores}

En TS esta lucha en defensa de los derechos humanos y la justicia social se hace desde el reconocimiento de la existencia de elementos que perpetúan las situaciones de desigualdad y exclusión que viven las personas, grupos y comunidades. Ante ello, se destaca la importancia de reflexionar sobre estos elementos, así como el desarrollo de estrategias para superarlos (IFSW, 2014). Para afrontar este reto del TS, la MC permite analizar no solo los elementos que perpetúan las situaciones de desigualdad y exclusión social sino también aquellos elementos que posibilitan el cambio social (C. Pulido, Elboj, Campdepadrós, \& Cabré, 2014).

El análisis de la información en la MC siempre incorpora las dimensiones exclusoras y transformadoras. De este modo, esta metodología posibilita dar un paso más en la investigación dando respuesta a necesidades de los diferentes colectivos y agentes sociales (Redondo-Sama, Pulido-Rodríguez, Larena, \& de Botton, 2014; Tellado, López-Calvo, \& Alonso-Olea, 2014), ofreciendo vías y estrategias para el cambio social.

\section{Participación de la comunidad}

Con el objetivo de generar cambio social, en TS una gran parte del conocimiento y evidencias científicas se construye juntamente con las personas, grupos y comunidades a partir del diálogo, permitiendo de este modo obtener información sobre los contextos en los que viven éstos y las barreras ante las que se enfrentan (IFSW, 2014). La Declaración Global de los Principios Éticos del TS especifica el deber del TS de promover el 
derecho de todas las personas a participar e implicarse en todos los aspectos de las acciones y decisiones que les atañen y su a tomar sus propias decisiones (IFSW, 2018). Asimismo, el artículo 27 de la Declaración de los Derechos Humanos, una declaración central en el TS, establece que toda persona tiene derecho a "participar en el progreso científico y en los beneficios que de él resulten" (Asamblea General de la ONU, 1948). En esta línea, la MC reconoce a los individuos como agentes con capacidad para entender el mundo, contribuir a generar conocimiento y transformar su contexto social (A. Gómez et al., 2019), siendo una de sus bases el diálogo igualitario e intersubjetivo entre la persona investigadora y los agentes sociales y los miembros de la comunidad (J. Gómez et al., 2006).

Estos elementos quedan evidenciados en los postulados de la MC y se ven reflejados en la organización de la investigación, en la que el diálogo se mantiene durante toda la investigación y en él, el investigador aporta el conocimiento científico y los colectivos y los agentes sociales su inteligencia cultural (Gómez et al., 2011). De este modo, se construye nuevo conocimiento con las personas y comunidades a partir del diálogo igualitario, respetando sus conocimientos. Este diálogo permite que haya un empoderamiento de las personas más vulnerables durante el proceso de investigación, lo que puede ser un desencadenante para mayores transformaciones sociales desde abajo (Aubert, 2011). Asimismo, el diálogo igualitario durante toda la investigación permite que todos los colectivos sean incluidos en todas las etapas de la investigación (A. Flecha, 2015). De este modo, la investigación se centra en las necesidades reales de las personas y comunidades, también de aquellas que tradicionalmente han sido excluidas de los espacios de toma de decisiones (Brown et al., 2013).

\section{Implicaciones para el Trabajo Social}

Ante el giro dialógico de la sociedad, cada día en más espacios las relaciones basadas en el diálogo y el consenso están substituyendo a las autoridades tradicionales (R. Flecha et al., 2004), aumentando, de este modo, las posibilidades de transformar las sociedades desde diferentes ámbitos. En este contexto, la ciudadanía está pidiendo decidir sobre su vida y sobre el gobierno de las sociedades en las que viven. Para poder decidir, 
es necesario no solo disponer de información sobre las causas e implicaciones de las situaciones sociales que padecen sino también aquellas estrategias que han evidenciado que funcionan para darles respuesta, como las Actuaciones de Éxito que ofrecen alternativas de transformación (Elboj Saso, 2014). Ante este contexto, se constata la importancia de disponer de metodologías de investigación que, por un lado, aporten evidencias útiles para la transformación y el cambio social y, por el otro, ofrezcan respuesta a las demandas de participación de la sociedad, también durante el proceso de investigación.

Este hecho es especialmente relevante con colectivos que tradicionalmente han quedado excluidos de la toma de decisiones y que deben afrontar a graves situaciones de desigualdad y exclusión social, como la comunidad gitana. Esta situación queda evidenciada en recientes informes, como es el caso de A persisting concern: antigypsism as a barrier to Roma inclusion, en el que se analizan y exponen las situaciones de desigualdad y exclusión que vive esta comunidad con relación a cinco ejes: educación, pobreza, ocupación, salud y discriminación, acoso y delitos de odio (European Union Agency for Fundamental Rights, 2018).

Ante la situación de exclusión que vive la comunidad gitana, el TS no puede quedarse estático. Es por esta razón que es necesario disponer de metodologías que nos permitan realizar investigaciones con impacto social y que concuerden con los principios y objetivos del TS. La MC ha evidenciado su impacto con los colectivos más vulnerables (A. Gómez et al., 2019), siendo recomendada como enfoque apropiado para superar sus situaciones de exclusión (R. Flecha \& Tellado, 2015). Asimismo, esta metodología ha demostrado su idoneidad para realizar investigaciones con la comunidad gitana, alcanzando un gran impacto político con el Proyecto WORKALÓ (Macías \& Redondo, 2012; Sordé et al., 2013) y social con el Proyecto INCLUD-ED (Melgar, Larena, Ruiz, \& Rammel, 2011). Además, los tres elementos identificados y analizados en el artículo sitúan a la MC como una metodología de investigación adecuada no solo en el contexto social actual sino también en relación a los objetivos y principios del TS.

Partiendo de las aportaciones de la MC al TS, las implicaciones del uso de esta metodología en la investigación en TS permiten disponer de evidencias para la práctica profesional, contribuyendo a reducir la brecha 


\section{Plaja Viñas - Aportaciones de la Metodología Comunicativa}

aún existente entre la investigación y la práctica, aumentando de esta manera, el impacto y el estatus del TS.

En lo relativo a la primera aportación de la MC al TS, las Actuaciones de Éxito son una vía para superar la brecha entre la investigación y la práctica en TS. Las Actuaciones de Éxito aportan los principios universales que posibilitan el éxito de las intervenciones, lo que permite que puedan ser usadas en diferentes contextos y colectivos (R. Flecha \& Soler, 2014). Además, al ser utilizadas, su implementación no se realiza a ciegas, sino que se realiza mediante el diálogo con los end-users (Elboj Saso, 2014).

En segundo lugar, en todas las áreas de la ciencia se está viviendo un giro hacia el impacto social de la investigación, es decir, a que la investigación contribuya a alcanzar los objetivos establecidos por la sociedad, como los Objetivos de Desarrollo Sostenible de las Naciones Unidas (United Nations, 2015). Ante este requerimiento, el TS se encuentra en una posición privilegiada que se ve reflejada en, por un lado, que tal como expone la definición de TS elaborada por la International Federation of Social Workers, el cambio social conforma la razón de ser de la profesión y disciplina (IFSW, 2014). Por otro lado, la posibilidad de tener contacto directo con los colectivos y comunidades que se están investigando, puesto que son los sujetos de la intervención social.

Finalmente, ante un contexto que en los últimos años está priorizando el impacto social de la investigación, la posición privilegiada del TS ante este requerimiento se presenta como una oportunidad no solo para reducir la brecha entre investigación y práctica sino también para aumentar el estatus científico del TS. En ocasiones, el TS ha sido considerado como una disciplina de segundo orden que aplica conocimientos de otras disciplinas (Fong, 2012; Shaw, Arksey, \& Mullender, 2006). Ante este contexto, la investigación se presenta como vía para un mayor estatus académico (Bywaters, 2007). Realizar investigaciones con una metodología reconocida internacionalmente por organismos como la Comisión Europea como metodología de investigación especialmente apropiada para trabajar con colectivos vulnerables por su orientación a la transformación social ( $R$. Flecha \& Tellado, 2015), posibilita no solo reducir la brecha entre la investigación y la práctica sino también contribuir al reconocimiento de la investigación en TS y, por lo tanto, a aumentar su estatus científico. 


\section{Conclusiones}

A partir del análisis de las contribuciones y el encaje de la MC en la investigación en TS, se evidencia como ante la brecha existente entre la investigación y la práctica en TS, el uso de la MC puede contribuir a reducirla, aumentando el impacto social de la investigación en TS y la eficiencia de sus intervenciones.

Asimismo, la MC destaca no solo por su orientación al impacto social sino también por la concordancia que existe entre esta metodología y los principios y objetivos del TS (IFSW, 2014; IFSW, 2018), posibilitando disponer de evidencias científicas para la práctica profesional elaboradas conjuntamente con las personas y comunidades más vulnerables. Esta metodología se presenta, por lo tanto, como especialmente adecuada en investigaciones con la comunidad gitana. Esta comunidad continúa viviendo graves situaciones de desigualdad y exclusión social ante las que desde el TS es necesario realizar investigaciones orientadas al cambio social, superando investigaciones que perpetúan las situaciones de exclusión.

Nos encontramos en un contexto en el que cada vez más se valora el impacto social de la investigación. Ante esta situación, la MC posibilita el impacto social de la investigación en TS, aumentando, de esta manera, el estatus científico de la disciplina.

Teniendo en cuenta lo expuesto y ante el impacto social de las investigaciones realizadas mediante la MC y su concordancia con el TS, se evidencia la importancia de continuar realizando investigaciones que nos permitan identificar y analizar Actuaciones de Éxito en esta disciplina. Esto contribuirá a la lucha por el cambio social en base a los derechos humanos y la justicia social.

\section{Bibliografía}

Addams, J. (2014). Veinte años en Hull House (1. ${ }^{\mathrm{a}}$ ed.). Murcia: edit.um. Aiello, E., \& Joanpere, M. (2014). Social Creation. A New Concept for Social Sciences and Humanities. International and Multidisciplinary 
154 Plaja Viñas - Aportaciones de la Metodología Comunicativa

Journal of Social Sciences, 3(3), 297-313. doi:

10.4471/rimcis.2014.41

Aiello, E., Puigvert, L., \& Schubert, T. (2018). Preventing violent

radicalization of youth through dialogic evidence-based policies.

International Sociology, 33(4), 435-453. doi:

$10.1177 / 0268580918775882$

Asamblea General de la ONU. (1948). Declaración Universal de los

Derechos Humanos.

Aubert, A. (2011). Moving beyond social exclusion through dialogue.

International Studies in Sociology of Education, 21(1), 63-75. doi:

10.1080/09620214.2011.543854

Brown, M., Gómez González, A., \& Munté Pascual, A. (2013). Procesos dialógicos de planificación de los servicios sociales: el proceso de cambio en los barrios de La Milagrosa y La Estrella (Albacete).

Scripta Nova. Revista Electrónica de Geografía y Ciencias Sociales, 17(427).

Bywaters, P. (2007). Learning from Experience: Developing a Research

Strategy for Social Work in the UK. British Journal of Social Work, 38(5), 936-952. doi: 10.1093/bjsw/bcm070

Drisko, J. W., \& Grady, M. D. (2015). Evidence-Based Practice in Social

Work: A Contemporary Perspective. Clinical Social Work Journal, 43(3), 274-282. doi: 10.1007/s10615-015-0548-z

Elboj Saso, C. (2014). In the Path to Regaining Social Sciences Legitimacy through Public Sociology. International and Multidisciplinary Journal of Social Sciences, 3(2), 158-181. doi: 10.4471/rimcis.2014.35

European Commission. (2011). Added value of Research, Innovation and Science portfolio [online]. Retrieved from:

http://europa.eu/rapid/press-release_MEMO-11-520_en.htm

European Union Agency for Fundamental Rights. (2018). A persisting concern: antigypsism as a barrier to Roma inclusion. Retrieved from: https://fra.europa.eu/sites/default/files/fra_uploads/fra-2018anti-gypsyism-barrier-roma-inclusion_en.pdf 
Flecha, A. (2015). Isabel, From Adult Learner to Community Activist. Qualitative Inquiry, 21(10), 865-871. doi:

$10.1177 / 1077800415611693$

Flecha, R., \& Soler, M. (2013). Turning difficulties into possibilities:

Engaging Roma families and students in school through dialogic learning. Cambridge Journal of Education, 43(4), 451-465. doi: 10.1080/0305764X.2013.819068

Flecha, R., Vargas, J., \& Dávila, A. (2004). Metodología comunicativa de investigación y cohesión social en Europa. Lan-Harremanak, Revista de Relaciones Laborales: Minorías culturales y nuevas tecnologías, 23-33.

Flecha, R. (Ed.). (2015). Successful Educational Actions for Inclusion and Social Cohesion in Europe. doi: 10.1007/978-3-319-11176-6

Flecha, R., Soler-Gallart, M., \& Sordé, T. (2015). Europe must fund social sciences. Nature, 528(7581), 193-193. doi: 10.1038/528193d

Flecha, R., \& Soler, M. (2014). Communicative Methodology: Successful actions and dialogic democracy. Current Sociology, 62(2), 232-242. doi: $10.1177 / 0011392113515141$

Flecha, R., \& Tellado, I. (2015). Metodología comunicativa en educación de personas adultas. Cadernos CEDES, 35(96), 277-288. doi: 10.1590/CC0101-32622015723765

Fong, R. (2012). Framing Education for a Science of Social Work: Missions, Curriculum, and Doctoral Training. Research on Social Work Practice, 22(5), 529-536. doi: 10.1177/1049731512452977

Garcia Yeste, C., Gairal Casado, R., Munte Pascual, A., \& Plaja Vinas, T. (2018). Dialogic literary gatherings and out-of-home child care: Creation of new meanings through classic literature. CHILD \& FAMILY SOCIAL WORK, 23(1), 62-70.

Garcia Yeste, C., Morlà Folch, T., \& Ionescu, V. (2018). Dreams of Higher Education in the Mediterrani School Through Family Education. Frontiers in Education, 3, 79. doi: 10.3389/feduc.2018.00079

Gómez, A., \& Munté, A. (2015). Communicative methodology of research and Romaní migrant women in Spain: a process of social change. En Critical and Creative Research Methodology in Social Work (pp. 6174). 
Gómez, A., Padrós, M., Ríos, O., Mara, L.-C., \& Pukepuke, T. (2019). Reaching Social Impact Through Communicative Methodology. Researching With Rather Than on Vulnerable Populations: The Roma Case. Frontiers in Education, 4, 9. doi:

10.3389/FEDUC.2019.00009

Gómez, A., Puigvert, L., \& Flecha, R. (2011). Critical Communicative Methodology: Informing Real Social Transformation Through Research. Qualitative Inquiry, 17(3), 235-245. doi:

10.1177/1077800410397802

Gómez, A., \& Díez-Palomar, J. (2009). Metodología Comunicativa Crítica:

Transformaciones y Cambios en el S. XXI. Teoría de la Educación.

Educación y Cultura en la Sociedad de la Información, 10(3), 103118.

Gómez, J., Latorre, A., Sánchez, M., \& Flecha, R. (2006). Metodología comunicativa crítica. Barcelona: El Roure

Gray, M., Sharland, E., Heinsch, M., \& Schubert, L. (2015). Connecting

Research to Action: Perspectives on Research Utilisation. British

Journal of Social Work, 45(7), 1952-1967. doi: 10.1093/bjsw/bcu089

International Federation of Social Workers. (2014). Global Definition of

Social Work. Retrieved from: http://ifsw.org/policies/definition-ofsocial-work/

International Federation of Social Workers. (2018). Global Social Work

Statement of Ethical Principles. Retrieved from:

https://www.ifsw.org/global-social-work-statement-of-ethicalprinciples/

Macías, F., \& Redondo, G. (2012). Pueblo gitano, género y educación: investigar para excluir o investigar para transformar. International Journal of Sociology of Education, 1(1), 71-92. doi:

10.4471/rise.2012.04

Melgar, P. (2015). Luis, From Ex-Prisoner to Neighborhood Representative and Interlocutor With Public Administrations. Qualitative Inquiry, 21(10), 872-878. doi: 10.1177/1077800415611694

Melgar, P., Larena, R., Ruiz, L., \& Rammel, S. (2011). How to Move from Power-based to Dialogic Relations? Lessons from Roma Women. European Journal of Education, 46(2), 219-227. 
Munte, A., Serradell, O., \& Sorde, T. (2011). From Research to Policy:

Roma Participation Through Communicative Organization.

Qualitative Inquiry, 17(3), 256-266. doi:

$10.1177 / 1077800410397804$

Munté, A., \& Vicente Zueras, I. De. (2012). El \&quot;Sueño de

Barrio\&quot; ¿Un nuevo modelo de Trabajo Social? Revista

Katálysis, 15(2), 254-261. doi: 10.1590/S1414-49802012000200011

Puigvert, L. (2012). The Dialogic Turn: Dialogue or Violence?

International and Multidisciplinary Journal of Social Sciences, 1(1),

78-96. doi: 10.17583/rimcis.2012.330

Pulido, C., Elboj, C., Campdepadrós, R., \& Cabré, J. (2014). Exclusionary and Transformative Dimensions Communicative Analysis Enhancing Solidarity Among Women to Overcome Gender Violence.

Qualitative Inquiry, 20(7), 889-894. doi:

$10.1177 / 1077800414537212$

Pulido, M. Á., \& Munté i Pascual, A. (2014). El papel del trabajo social en el Sueño de Barrio. RASE. Revista de la Asociación de Sociología de la Educación, 2(3), 56-65.

Redondo-Sama, G., Pulido-Rodríguez, M. A., Larena, R., \& de Botton, L. (2014). Not Without Them: The Inclusion of Minors' Voices on Cyber Harassment Prevention. Qualitative Inquiry, 20(7), 895-901. doi: $10.1177 / 1077800414537214$

Richmond, M. E. (2005). Diagnóstico social. Madrid: Consejo General de Colegios Oficiales de Diplomados en Trabajo Social y Asistentes Sociales/Siglo XXI de España Editores, S.A.

Rios, O. (2013). Sociocultural Transformation \&amp; Development. Good

Practices or Successful Actions. Multidisciplinary Journal of

Educational Research, 3(2), 173-199. doi: 10.4471/remie.2013.11

Rubin, A. (2014). Bridging the Gap between Research-Supported

Interventions and Everyday Social Work Practice: A New Approach.

Social Work, 59(3), 223-230. doi: 10.1093/sw/swu023

Rubin, Allen. (2015). Efforts to Bridge the Gap Between Research and

Practice in Social Work: Precedents and Prospects: Keynote Address at the Bridging the Gap Symposium. Research on Social Work Practice, 25(4), 4408-4414. doi: 10.1177/1049731514535852 
158 Plaja Viñas - Aportaciones de la Metodología Comunicativa

Shaw, I. F., Arksey, H., \& Mullender, A. (2006). Recognizing Social Work. British Journal of Social Work, 36(2), 227-246. doi:

10.1093/bjsw/bch251

Sorde, T., \& Mertens, D. M. (2014). Mixed Methods Research With Groups at Risk. Journal of Mixed Methods Research, 8(3), 207-211. doi: $10.1177 / 1558689814527916$

Sordé, T., Flecha, R., \& Mircea Alexiu, T. (2013). El pueblo gitano: una identidad global sin territorio. Scripta Nova revista electrónica de geografía y ciencias sociales, XVII(427 (3)).

Steens, R., Van Regenmortel, T., \& Hermans, K. (2018). Beyond the Research-Practice Gap: The Development of an Academic Collaborative Centre for Child and Family Social Work. The British Journal of Social Work, 48(6), 1611-1626. doi: 10.1093/bjsw/bcx126

Tellado, I., López-Calvo, L., \& Alonso-Olea, M. J. (2014). Dialogic Design of Qualitative Data Collection for Researching the Mirage of Upward Mobility. Qualitative Inquiry, 20(7), 856-862. doi:

10.1177/1077800414537207

Thyer, B. A. (Ed.). (2010). The Handbook of Social Work Research Methods. Thousand Oaks: SAGE Publications, Inc.

United Nations. (2015). Resolution adopted by the General Assembly on 25 September 2015. In Transforming Our World: The 2030 Agenda for Sustainable Development. Retrieved from:

http://www.un.org/ga/search/view_doc.asp?symbol=A/RES/70/1\&La $\mathrm{ng}=\mathrm{E}$

Valls, R., \& Padrós, M. (2011). Using Dialogic Research to Overcome Poverty: from principles to action. European Journal of Education, 46(2), 173-183. doi: 10.1111/j.1465-3435.2011.01473.x

Valls, R., Puigvert, L., Melgar, P., \& Garcia-Yeste, C. (2016). Breaking the Silence at Spanish Universities: Findings From the First Study of Violence Against Women on Campuses in Spain. Violence Against Women, 22(13), 1519-1539. doi: 10.1177/1077801215627511

Teresa Plaja Viñas es Investigadora predoctoral en formación FPU en la Universidad de Barcelona

Dirección de contacto: teresa.plaja@ub.edu 